\title{
Needs of occupational exposure sampling strategies for compliance and epidemiology
}

\author{
K Gardiner
}

\begin{abstract}
Although a great deal of occupational exposure data is collected, it is probably insufficient to truly answer the question of legislative compliance, ill directed in terms of real workplace risks, and is of little subsequent use for epidemiological research. This paper is an attempt to summarise the more important components and requirements of a sampling strategy, and it is therefore aimed at those with this responsibility. Perhaps, all too frequently, the more esoteric nature of these issues and their research means that they are published in journals outside the normal sphere of readership, or when it is within that sphere the quantity of statistical nomenclature and content makes it too daunting to attempt to read. By simplifying and summarising, this paper is intended to help justify a change in the sampling programme and to initiate debate.
\end{abstract}

(Occup Environ Med 1995;52:705-708)

Keywords: sampling; compliance; epidemiology

The collection of occupational exposure data is time consuming, expensive, and unless taken as part of a coherent sampling strategy, often of little use. It is therefore essential that any measurements are taken after a number of fundamental issues have been considered; and it is this logistic approach that defines a sampling strategy and ensures that the desired confidence in the accuracy and precision of the measured concentrations are achieved.

It must be the main aim of all occupational health professionals to reduce ill health at work by the quickest and most effective means possible. I think it is therefore encumbent on occupational hygienists to ask themselves whether it is necessary to take any measurements at all, if a situation is obviously wrong and readily rectifiable without the taking of measurements then we are duty bound to do so without this additional delay. Occupational exposure data is collected for a variety of different reasons but the two main ones are to ascertain compliance with regulatory or in house occupational exposure limits (OELs) and to provide measures of exposure for epidemiological studies. This paper aims to identify the issues that must be considered for both, and where appropriate compare and contrast the different requirements. Also, although focused on occupational hygiene sampling the questions and approach are similar for all occupational health professionals where there is a need to sample.

\section{Exposure variability}

It would simplify greatly the task of the occupational hygienist if everyone doing the same job were exposed to the same contaminants, at the same concentration, over the same period of time, day after day regardless of shift, production rate, etc. Unfortunately, however, the world is not like that and the variability of exposure is potentially very great and dependent upon such factors as: the nature, density and intensity of activity, the process or people, the contaminants of interest, and environmental components such as temperature, windspeed, wind direction, humidity, etc. This means that the resultant large variability within and between workers makes the sampling strategy absolutely crucial.

\section{Structure of sampling strategy}

As mentioned previously, there are several questions that form the basis of any sampling strategy and these are: what to measure; how to sample; whose exposure should be measured; where to collect the sample; when to measure; how long to sample for, and how many measurements or readings to take? The following sections will consider each of these in turn.

\section{What to measure?}

As it is rare for only one substance to be used in industrial settings the first question to be answered in the assessment of compliance is which, of potentially many contaminants, should be measured. Three main options exist: (a) all, or many, of the contaminants (where the additive formula may be needed); (b) the mixture as a whole (such as rubber or welding fume); or (c) reference or surrogate 
substances (such as a polycyclic aromatic hydrocarbon (PAH) which stays in constant ratio with all of the others in a mixture).

In epidemiological research it is necessary to identify the biologically relevant measure of exposure - that is, the aeteological agent. This may be the same as that required for compliance testing but examples exist, such as that for bakers' asthma where it would be preferable to measure the level of airborne $a$-amylase (a dough improver) rather than the concentration of total inhalable dust.

\section{How to sample?}

There is not space within this paper to reiterate the multitude of different techniques for measuring contaminants in the workplace. Not foresaking practical issues with the equipment, the sampling and analytical techniques selected should meet the requirements of the sampling strategy and not vice vers $a^{1}$ - that is, that where necessary the accuracy, precision, specificity, and sensitivity are maximised accordingly.

\section{Whose exposure should be measured?}

In sampling strategies for both compliance and epidemiological purpose the decision of who to sample is vital. In the past, compliance testing strategies have focused on "worst case scenarios" whereby people undertaking the jobs likely to give rise to the highest exposure or an intrinsically "dirty worker" are sampled. ${ }^{2}$ This was done on the basis that if this exposure was less than the OEL, then by default so would exposure in all other situations. Unfortunately, this biased selection process was driven by legislative requirements to ascertain the probability that a person exceeded the OEL on a particular day ${ }^{3-5}$ but these data are profoundly limited for other purposes.

The current promulgated technique is for groups of workers with common exposure to be formed either prospectively or retrospectively and for a subset of these to be sampled randomly. Common exposure means that the group should be exposed to the same substances and that each of the exposure distributions for the individual workers have the same means (SDs) - this is often referred to as homogeneity.

Grouping of prospective employees relies on the ability of the occupational hygienist to assign individual workers to a group based on observations such as similarity of tasks, contaminants, and environment (process equipment and controls). ${ }^{6}$ A proportion of workers from within each group should be selected randomly and then sampled with the data then assessed for homogeneity. Environments where populations generate data that meet this definition of homogeneity are almost unheard of and so a more "workable" definition is required. The Health and Safety Executive in the United Kingdom have suggested a crude but useful rule, that is: if an worker's exposure is less than half, or greater than twice, the group mean then they should be reassigned to another group. ${ }^{7}$ Another definition of a homogeneous or monomorphic group is described in the retrospective grouping of employees.

\section{Retrospective grouping of employees}

As it is not always possible to assign workers to the correct groups simply on the basis of observation, especially those involved in maintenance or non-routine tasks, it may be preferable to randomly sample everyone. This procedure gains in significance if it is possible to undertake at least two sets of repeat measurements on those sampled, and helps to identify the components of variability of exposure - that is, total distribution, and distributions within and between workers. The groups of workers can now be calculated on the basis of the exposure data itself (concentration and variability). A grouping is deemed to be monomorphic if $95 \%$ of the individual mean exposures lie within a factor of 2 . This implies that the ratio of the $97 \cdot 5$ th percentile to the $2 \cdot 5$ th percentile $\left(R_{0.95 B}\right)$ is not greater than 2 , which equates to a between worker geometric $(\mathrm{SD})\left(\sigma_{\mathrm{B}}, \mathrm{B}\right)$ of $\leqslant 1 \cdot 2 .{ }^{1}$ If groups used in epidemiology are not homogeneous, which could cause insufficient contrast so that the exposure distribution of two groups overlap-that is, contrast-then misclassification can occur. This tends to attenuate the exposure-response relation -although it is more important for the hygienist to ensure that even if the groups are not homogeneous they do not overlap-wherein the estimate of the relative risk at a certain exposure level will be unbiased but lacking precision. ${ }^{9}$

In the main, grouping of prospective employees has been used for compliance purposes with the retrospective technique used for epidemiology. In recent years, however, it has been thought that a combination of the two techniques can serve the needs of both compliance and epidemiology.

\section{Where to collect the sample}

To a great extent sample location is dictated by legislative and practical requirements. Most OELs are set for personal exposure, which is usually defined as the microenvironment around the individual worker's breathing zone $(20-30 \mathrm{~cm}$ from nose or mouth). The lapel is the most common location, although for substances with a high degree of thermal buoyancy a sampler held next to the nose or mouth by cranial caps (such as for welding fume) may be preferable.

Despite poor correlations between exposure measured at personal and static locations, there are several substances where the specified technique is for a static location (such as cotton dust and vinyl chloride monomer in the United Kingdom). Most of the situations in which these measurement are used are dictated by the bulkiness of the equipment or the 
desire to have a fixed location such as for assessment over time of control measures.

\section{When to measure?}

Processes can be split into three main types; continuous, cyclic, or random; with most major processes being made up of different proportions of all three-for example, in a chemical factory the production is continuous, the packaging is cyclic, and the reactive maintenance random. If a worker's job involves just one of these types of process then both cyclic and random exposures will vary considerably but will be more stable for a continuous process. This exposure variability will be even greater if a worker is involved with two or more types of process. Clearly, regardless of whether measuring for compliance or epidemiological purposes, there is a need to be aware of this variability and to sample at such times as to reflect this most accurately. If a random sampling programme is used care needs to be taken to ensure that sufficient samples are taken so that rare tasks are likely to be included.

\section{How long to sample for?}

This is an area of great potential divergence between the requirements of compliance and epidemiology. Compliance testing requires the comparison of exposure with legislative airborne standards of which there are, in the main, two time weighted average (TWA) reference periods-eight hours and 15 minutes (in Germany it varies from five to 60 minutes). Although, it is not necessary to sample for these exact durations as within the reference time there may be periods of known exposure (zero or some other value). This thereby facilitates greater precision and accuracy to be gained for the periods that are evaluated. It is then possible to calculate the TWA exposure relative to the control periods.

Epidemiological evaluation poses greater problems as it is necessary to have some knowledge of the rate at which the contaminant causes a biological effect-that is, if the substances have an acute effect (seconds to hours) then the duration of sampling must be able to reflect this variability within a shift whereas if the effect is chronic then a more appropriate duration may be a weekly, monthly, annual, average, or lifetime dose.

For substances known to cause an immediate effect on say mucous membranes, such as sulphur dioxide, ceiling values are quoted in some countries. Instantaneous measurement of these contaminants is difficult as instrumentation only exits for a small number of substances, and the accuracy, precision, and specificity are often limited. Epidemiologically the problem may be compounded further by issues of the exposure profile. It has been postulated that it is in some way the "peakiness" of the exposure (profile) to sensitisers that causes the sensitisation rather than the dose and much research needs to be undertaken before these questions can be answered. ${ }^{10}$
How many measurements or readings?

It should be obvious that in any given work situation there is likely to be a lot of variance within or between workers. This means, therefore, that simply taking a small number of samples from a few volunteers on one day is dangerously inappropriate. Everyday constraints, such as time and money, mean that in large groups (> 10 people doing the same job) it is not always possible to take duplicate samples from the whole group. For compliance, a sufficient number of samples need to be taken to reach a decision about the possibility of overexposure and for epidemiology precision in the estimate of a worker's or group's average exposure is required.

Several techniques have been proposed to decide the number of samples to be taken. ${ }^{31112}$ These do not specify that each worker should be sampled at least twice (preferably the same number of repeats on each person), and so they facilitate the calculation of within and between worker variances by the use of a balanced one way random effect analysis of variance (ANOVA). Kromhout and co-workers used a total of 10 samples (five people sampled twice) as a minimum, ${ }^{13}$ although with these numbers comparison of the group mean with the OEL will lack statistical power and so more than 20 (five people four times or 10 people twice) is recommended. If the number of samples and the resultant exposure indicate that the question of overexposure cannot be resolved, then it is necessary to resample choosing to take either the same number of repeat samples from extra people or to take repeat measurements on all of those already sampled and by so doing ensure that the numbers of repeats on each person remain balanced. Little additional benefit is gained by taking more than six repeat samples. If overexposure does exist then review of the variances within and between workers should indicate where control measures should be focused; engineering controls for high variance within workers (day to day) and administrative (behavioural) controls for high variance between workers.

In epidemiology, the numbers of samples needed often relies on detailed information about the partitioning of the exposure variability before a study formally starts. For example, in studies where everyones' exposure is measured then the number of repeat measurements per worker can be calculated (along with prediction of the bias in the regression coefficient) by use of the within to between worker variance ratio $(\lambda)::^{14}$

$$
\begin{array}{ll}
\beta^{\star} & =\beta\left(1+\lambda \cdot \mathrm{n}^{-1}\right)^{-1} \\
\text { where } \beta^{\star} & =\text { observed regression coefficient } \\
\beta & =\text { true regression coefficient } \\
\lambda & =\text { variance ratio }={ }_{\mathrm{ww}} S^{2}{ }_{\mathrm{y}}\left(\mathrm{Bw}_{\mathrm{w}} \mathrm{S}_{\mathrm{y}}\right)^{-1} \\
{ }_{\mathrm{ww}} \mathrm{S}_{\mathrm{y}}^{2} & =\text { within worker variance } \\
{ }_{\mathrm{Bw}} \mathrm{S}_{\mathrm{y}}^{2} & =\text { between worker variance } \\
\mathrm{n} & =\text { number of measurements per } \\
& \text { person }
\end{array}
$$

As it is the variance ratio that is the important measure in determining the amount of the underestimation of the empirical regression coefficient, the absolute values of either of the 
two components (within and between workers) is less relevant (the variance within workers could be large but the variance between workers even larger). If a study is proposed in a population with a large variance ratio or relatively large exposure variability within workers then it is possible to calculate the number of repeat samples if knowledge of the bias in the regression coefficient is known or set: ${ }^{1415}$

$$
\mathrm{k}=\left(\mathrm{p} .(1-\mathrm{p})^{-1}\right) \cdot \lambda
$$

where $\mathrm{k}=$ number of repeat measurements for each person

$\mathrm{p}=$ bias in regression coefficient $\beta^{\star} / \beta$

$\lambda=$ variance ratio

For example, if $\lambda=6$ then at least 54 measurements are required on each worker to reduce the relative bias in the regression coefficient to a maximum of $10 \%(P=0.9) .{ }^{15}$ If this effort is too great, then it is possible to sample subsets of the population, calculate the variance, within and between workers and then make a correction for the attenuation of the exposure-response relation. ${ }^{8}$

If an initial grouping strategy is used the numbers of samples required is most important in terms of its influence on the size of the SEM exposure (precision). The variance within a group (homogeneity) and between groups (contrast) is also related to number of samples. ${ }^{1516}$ Obviously, an optimal grouping would be where the groups are homogeneous, great contrast exists between the groups and the mean exposure in each group is precise. Unfortunately, however, these are rarely compatible due to the practical realities of a limited number of samples; small homogeneous groups have poor contrast and precision and big groups have greater precision and contrast but less homogeneity. The relative efficiency of either personal or grouped assessments should be compared and contrasted on the basis of power calculations and costs. ${ }^{17} 18$

\section{Miscellaneous issues and summary}

Most exposure sampling is undertaken for compliance testing. If the issues identified and considered within this paper are acted upon at the time when compliance programmes are developed, then it is thought that not only will the compliance data be better able to provide the information required but also that after its immediate use it would be a most valuable legacy if it were needed for retrospective exposure assessments. If the forethought of any epidemiological use is built into all sampling strategies, which I think it should be, then there are several other issues that should be considered. In any survey report, the actual purpose or philosophy behind the survey and design should be explained explicitlyprobably in the introduction or discussion. Frequently, survey reports identify people by job titles but over the years the actual tasks that make up a job title changes radically but the title remains the same. It is therefore of great use if these are recorded along with the more modern requirements of job rotation where the durations of the different tasks involved would be most valuable. Akin to this is the need to provide detail about the actual tasks (and durations) undertaken during the period of sampling, ${ }^{19}$ especially how these relate to the range of activities and intensities usually experienced. A plan or layout of the workplace is also of use. Lastly, within this abbreviated list, is some feel for the change in measurement techniques over time and how this may have affected the results.

1 Rappaport SM. Assessment of long-term exposure to toxic substances in air. Ann Occup Hyg 1991;35:61-121.

2 Olsen E, Laursen B, Vinzents PS. Bias and random errors in historical data of exposure to organic solvents. Am Ind Hyg Assoc $¥ 1991 ; 52: 204-11$.

3 Leidel NA, Busch KA, Lynch JJ. Occupational exposure sampling strategy manual. Cincinnati: DHEW (NIOSH) sampling strategy manual.

4 Tuggle RM. Assessment of occupational exposure using one-sided tolerance limits. Am Ind Hyg Assoc $\mathcal{F} 1982 ; 43$. 338-46.

5 Selvin S, Rappaport SM, Spear R, Schulman J, Francis M A note on the assessment of exposure using one-sided tolerance limits. Am Ind Hyg Assoc f 1987;48:89-93.

6 Corn M, Esmen NA. Workplace exposure zones for classification of employee exposures to physical and chemical agents. Am Ind Hyg Assoc $¥$ 1979;40:47-57.

7 Health and Safety Executive. Monitoring strategies for toxic substances. London: Health and Safety Executive, HMSO, HSE Guidance Note EH42.

8 Heederick DJ, Miller BG. Weak associations in epidemiology: adjustment for exposure estimation error. Int $\mathcal{f}$ Epidemiol 1988;17:970-4.

9 Heederick D, Kromhout H, Burema J. Letter to the editor. Ann Occup Hyg 1991;36:671-3.

10 Gardiner K. Editorial: exposure profiles and respirator sensitizers. Occup Hyg 1995;1:243-5.

11 Rappaport SM, Selvin S. A method for evaluating the mean exposure from a lognormal distribution. Am Ind $\mathrm{Hy}$ Assoc $\mathcal{F}$ 1987;48:374-9.

12 Evans J, Hawkins NC. The distribution of student's $t$-statistic for small samples from loonormal exposure dis-

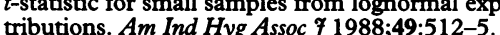

13 Kromhout H, Symanski E, Rappaport SM. A comprehensive evaluation of within-and between-worker composive evaluation of within-and between-worker components of occupational exposur.
Occup Hyg 1993;37:253-70.

14 Liu K, Stanler J, Dyer A, McKeever J, McKeever P. Statistical methods to assess and minimise the role of intra-individual variability in obscuring the relationship between dietary lipids and serum cholesterol. 7 Chron Dis 1978;31:399-418.

15 Boleij JSM, Buringh E, Heederick D, Kromhout $H$ Occupational hygiene of chemicals and biological agents. Amsterdam: Elsevier, 1995.

16 Kromhout H, Heederik D. Occupational epidemiology in the rubber industry: implications of exposure variability the rubber industry: implications

17 Philips AN, Smith GD. The design of prospective epidemiological studies: more subjects or better exposure miological studies: more subjects or better expo

18 White E, Kushi LH, Pepe MS. The effect of exposure variance and exposure measurement error on study sample size: implications for the design of epidemiologic studies. f Clin Epidemiol 1994;48:873-80.

19 Kromhout H, Swuste P, Beleij JSM. Empirical modellin of chemical exposure in the rubber-manufacturing industry. Ann Occup Hyg 1994;38:3-22. 